\title{
Simulation of dry and lubricated contacts in multi-body systems
}

\author{
Éric Quetel ${ }^{1,2, a}$, Johannes Müllers ${ }^{3}$, Daniel Coutellier $^{1}$ And Markus Hinterkausen ${ }^{2}$ \\ 1 LAMIH UMR CNRS 8530, Université de Valenciennes et du Hainaut Cambrésis, 59313 Valenciennes Cedex 9, France \\ 2 Robert Bosch GmbH, Corporate Research, CR/ARU1, Robert-Bosch-Platz 1, 70839 Gerlingen-Schillerhöhe, Germany \\ 3 Robert Bosch GmbH, Diesel Systems, DS/ETI1, Wernerstrasse 51, 70469 Stuttgart-Feuerbach, Germany
}

Received 25 May 2007, Accepted 24 January 2008

\begin{abstract}
The present paper investigates the modeling of counterformal elastohydrodynamic lubricated (EHL) contacts in multi-body system (MBS) environments. The aim is to obtain a representation of the dynamic behaviour of machine elements and characteristic values as the lubrication film thickness for lifetime calculations. Two contact models are presented for the study of cam-roller tappet and racewayroller systems. The first model is a one-dimensional representation of the EHL contact behaviour. It is based on the calculation of the central lubrication film thickness by means of dimensionless values (functions of the operating conditions) and provides a simplified model of the mixed lubrication. The second one, still under development, investigates the modeling of 3D dry and lubricated contacts. The fluid-structure interaction is here fully considered and a discretised form of the contact problem is solved using an iterative algorithm. This paper proposes, in the latter case, some perspectives and a discussion of its implementation.
\end{abstract}

Key words: lubrication / Elastohydrodynamic/ mixed lubrication / multi-body system / contact

\begin{abstract}
Résumé - Simulation de contacts secs et lubrifiés dans les systèmes multi-corps. L'article suivant concerne la modélisation de contacts élastohydrodynamiques entre semi-espaces dans les environnements de systèmes multi-corps dans le but d'obtenir une représentation du comportement dynamique de mécanismes complexes et d'évaluer les grandeurs caractéristiques, comme l'épaisseur de lubrifiant, nécessaires aux calculs de durée de vie. Deux modèles de contact élastohydrodynamique sont ainsi présentés pour l'étude de systèmes du type came-roulement ou piste-roulement. Le premier modèle, qui est une représentation unidimensionnelle du contact, s'appuie sur le calcul de la hauteur du film de lubrifiant au centre du contact, donnée à partir de coefficients sans dimension dépendant des conditions de fonctionnement. Il inclut une modélisation simplifiée du frottement mixte. Le second, encore en cours de développement, concerne la modélisation 3D de contacts secs et lubrifiés. L'interaction fluide-structure y est intégralement considérée et une solution du système discrétisé est adoptée. L'article contient, à cet effet, une discussion ainsi que quelques perspectives quant à son implémentation.
\end{abstract}

Mots clés : Lubrification / élastohydrodynamique / frottement mixte / système multi-corps / contact

\section{Introduction}

Elastohydrodynamically lubricated (EHL) contacts commonly occur in mechanical systems such as gears and cam-roller tappet systems. The calculated pressures of these contacts result from an interaction between the behaviour of the lubrication and the deformation of the structures. The study of this phenomenon is essential for optimizations and lifetime calculations of industrial applications. Due to the increasing complexity of these

\footnotetext{
${ }^{a}$ Corresponding author: eric.quetel@de.bosch.com
}

mechanisms, it becomes necessary to consider all interactions (contacts, joints...) of a system in order to obtain a complete description of the dynamic behaviour of each of its elements. However, the definition of contacts in multibody system (MBS) environments is often insufficient to represent the complexity of EHL contacts. Nowadays several methods exist to solve the EHL contact problem and then compute the contact pressure distribution and the lubrication film thickness.

EHL line and point contacts have been solved by Moes [1] with the introduction of dimensionless coefficients in order to compute the minimal and central 


\section{Nomenclature}

\begin{tabular}{|c|c|}
\hline$A$ & nominal contact area \\
\hline$A_{\text {asp }}$ & real contact area \\
\hline$A_{\mathrm{m}}$ & local nominal contact area \\
\hline$d$ & mutual approach between the two contacting bodies \\
\hline$E^{\prime}$ & reduced modulus of elasticity $\left(2 / E^{\prime}=\left(\left(1-\nu_{1}^{2}\right) / E_{1}\right)+\left(\left(1-\nu_{2}^{2}\right) / E_{2}\right)\right)$ \\
\hline$F_{\text {asp }}$ & mean contact force carried by the asperities \\
\hline$F_{\mathrm{e}}$ & normal contact force \\
\hline$F_{\mathrm{R}, \mathrm{B}}$ & boundary lubrication force \\
\hline$F_{\mathrm{R}, \mathrm{H}}$ & hydrodynamic friction force \\
\hline$h$ & lubricant film thickness \\
\hline$H_{\mathrm{C}}$ & dimensionless central film thickness \\
\hline$H_{\mathrm{EI}}$ & dimensionless central film thickness for elastic-isoviscous behaviour \\
\hline$H_{\mathrm{EP}}$ & dimensionless central film thickness for elastic-piezoviscous behaviour \\
\hline$H_{\mathrm{RI}}$ & dimensionless central film thickness for rigid-isoviscous behaviour \\
\hline$H_{\mathrm{RP}}$ & dimensionless central film thickness for rigid-piezoviscous behaviour \\
\hline$k$ & influence coefficients \\
\hline$l_{\text {eff }}$ & length of the line contact \\
\hline$L$ & dimensionless lubricant parameter (Moes) \\
\hline$M$ & dimensionless load parameter (Moes) \\
\hline$p$ & total contact pressure \\
\hline$p_{\text {asp }}$ & average contact pressure carried by the asperities \\
\hline$p_{K}$ & critical pressure \\
\hline$q_{x}, q_{y}$ & tangential traction in $x$ - and $y$-direction \\
\hline & residual \\
\hline$s_{x}, s_{y}$ & sliding distance in $x$ - and $y$-direction \\
\hline$u_{x}, u_{y}$ & grid mesh size in $x$ - and $y$-direction \\
\hline$U_{\mathrm{R}}$ & relative velocity in the contact \\
\hline$U_{\mathrm{S}}$ & sum of the surface velocities \\
\hline$w$ & underrelaxation factor \\
\hline$\alpha_{\text {asp }}$ & ratio of the real contact and the nominal contact area (Greenwood/Tripp) \\
\hline$\alpha_{\mathrm{C}}$ & ratio of the real contact and the nominal contact area (generalized model) \\
\hline$\alpha_{\mathrm{p}}$ & pressure viscosity coefficient \\
\hline$\beta_{\mathrm{GT}}$ & radius of curvature at the asperities \\
\hline$\delta_{x}, \delta_{y}$ & tangential displacement in $x$ - and $y$-direction \\
\hline$\zeta$ & Hertzian parameter \\
\hline$\eta$ & viscosity $\left(\eta=\eta_{0} \exp \left(\alpha_{\mathrm{P}} p\right)\right.$ according to Barus $)$ \\
\hline$\eta_{0}$ & viscosity at ambient pressure \\
\hline$\eta_{\mathrm{GT}}$ & effective density of the asperities \\
\hline$\Lambda$ & pressure correction \\
\hline$\mu_{\text {Bound }}$ & boundary lubrication coefficient \\
\hline$\rho_{\Sigma}$ & sum of the contacting bodies curvatures \\
\hline$\rho_{x}$ & sum of the contacting bodies curvatures of the $x$-direction \\
\hline$\sigma_{\mathrm{S}}$ & effective standard deviation of summits \\
\hline$\tau_{\text {Eyring }}$ & Eyring shear stress \\
\hline
\end{tabular}

lubrication film thickness as functions of several operating conditions, e.g. the surface velocity or the viscosity. This method is an extension of the principles introduced by Hamrock \& Dowson [2], which includes asymptotic behaviour for a larger applicability. The inverse method was first introduced by Dowson \& Higginson [3] for the solution of the line contact problems and then reworked by Evans \& Snidle [4] and Hou et al. [5] for point contacts. This method solves the complete EHL problem and provides after the solution of the Reynolds and Boussinesq equations a satisfactory description of the pressure distribution and film thickness for a good calculating duration. This method is, however, semi-empirical and may be unstable for lightly loaded cases. The multigrid method, described by Lubrecht \& Venner [6], is nowadays an excellent alternative to direct methods such as Newton-Raphson by offering a better convergence, stability and computation time. This method solves the discrete Reynolds and Boussinesq equations using several levels with different mesh densities.

The aim of this paper is to propose different adaptations of these methods to MBS environments in order to obtain a better dynamic representation of EHL contacts and provide the main characteristic values for lifetime studies. 


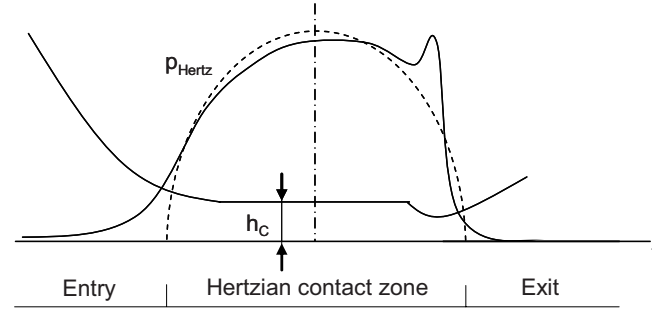

Fig. 1. Pressure distribution and film thickness of an EHL elliptic contact.

\section{One-dimensional EHL contact solution}

The following mathematical model aims the calculation of the dynamic behaviour of heavily loaded line contacts of cam-roller tappet or raceway-roller systems. During the MBS simulation, only a few contact characteristics are available. The system consists of rigid bodies and thus does not contain any deformation. Only the mutual approach between two contacting bodies and general kinematic values as body velocities are known. The MBS environment controls the location of the contact domain and the normal and tangential contact directions. The contact model is implemented as a user-subroutine which computes the contact forces and the lubrication film thickness. The fluid-structure interaction is calculated according to the elasticity theories of point and line contact $[7,8]$ and the theory of Moes [1]. A simplified mixed lubrication model is included to compute the friction force. It consists in the coupling of the Greenwood/Tripp theory [9] and the mathematical model of Teodorescu $[10,11]$.

\subsection{Lubricated contact conjunctions and mixed friction}

According to the heavy loads present in cam-roller contacts of high-pressure pumps, the pressure distribution of EHL (half-)elliptic contacts can be approximated to the elastic one, especially in the central contact area (Fig. 1). Thus, the normal contact force may be evaluated for a given mutual approach for the point contact as follows [7]:

$$
F_{\mathrm{e}}=E^{\prime} \sqrt{3}\left(\frac{2 d}{3 \zeta \rho_{\Sigma}^{1 / 3}}\right)^{3 / 2}
$$

and for the line contact by [8]:

$$
\begin{aligned}
d=\frac{4 F_{\mathrm{e}}}{\pi E^{\prime} l_{\mathrm{eff}}}\left[1.1931+\ln \left(\frac{l_{\mathrm{eff}} \rho_{x}}{2}\right)\right. & \\
& \left.-\frac{1}{2} \ln \left(\frac{2 F_{\mathrm{e}} \rho_{x}}{\pi E^{\prime} l_{\mathrm{eff}}}\right)\right]
\end{aligned}
$$

In Equations (1) and (2) body curvatures are fixed or determined by means of a spline interpolation at each simulation time step. The determination, not detailed here, of the normal contact pressure and nominal contact area is performed from the same elasticity theories. According to
Moes [1], the central lubrication film thickness may be calculated using two or three dimensionless coefficients. For the line-contact case, these coefficients are defined by:

$$
\begin{aligned}
& M=\frac{F_{\mathrm{e}} \rho_{x}}{E^{\prime} l_{\mathrm{eff}}}\left(\frac{E^{\prime}}{\eta_{0} U_{\mathrm{S}} \rho_{x}}\right)^{1 / 2} \text { and } \\
& L=\alpha_{\mathrm{P}} E^{\prime}\left(\frac{\eta_{0} U_{\mathrm{S}} \rho_{x}}{E^{\prime}}\right)^{1 / 4}
\end{aligned}
$$

Four interpolation functions are defined to describe the four asymptotic regimes of the lubrication: elasticisoviscous (EI), elastic-piezoviscous (EP), rigid-isoviscous $(\mathrm{RI})$, and rigid-piezoviscous (RP). These depend on the values of $M$ and $L$ according to various numerical and analytical results. The central film thickness is then calculated from a function fit formula of these regimes; see Appendix A for the line contact case. An estimation of these coefficients leads to the elastic-piezoviscous and elastic-isoviscous domains for the cam-roller contact under consideration which means that the elastic deformation as the piezo-viscosity cannot be neglected. Except the force, all necessary quantities for the calculation of the dimensionless coefficients are included in the input data or calculated during the simulation, e.g. the surface velocities.

The mixed lubrication is incorporated by means of the superposition of a hydrodynamic and a boundary lubrication model. In the case of heavily loaded cam-roller contacts, the hydrodynamic friction may be neglected. Thus, its representation is approximated to the viscous shearing of a Newtonian fluid:

$$
F_{\mathrm{R}, \mathrm{H}}=A \frac{\eta(p) U_{\mathrm{R}}}{h}\left(1-\alpha_{\mathrm{C}}\right)
$$

The boundary lubrication results from the superposition of friction due to local asperity contacts and from the shearing of an extremely thin lubricant film:

$$
F_{\mathrm{R}, \mathrm{B}} / A=\mu_{\text {Bound }} p+\tau_{\text {Eyring }} \alpha_{\mathrm{C}}
$$

The first term of the Equation (5) represents a Coulombtype law and consists of an empirical local friction coefficient multiplied by the contact force carried by the asperities. The second one results from the product of an empirical Eyring shear stress and the asperity contact area [10]. The coefficient $\alpha_{\mathrm{C}}$ is the ratio of the real asperity contact area on the total contact area. In the case of the heavily-loaded contacts, the real contact area may reach more than $50 \%$ of the total nominal contact area. Its calculation relies on a generalization of the Greenwood/Tripp theory [9]; see Appendix B.

The complete calculation procedure is presented in Figure 2. The three main phases are distinguished: the determination of the contact stiffness, respectively the elastic normal pressure or force, then the central lubrication film thickness and finally the friction forces. Two types of input data are defined: user inputs (USI) and dynamically calculated inputs (DCI). The first-ones are read at start and maintained for the whole simulation. The second-ones are determined at each time step of the MBS solver. 


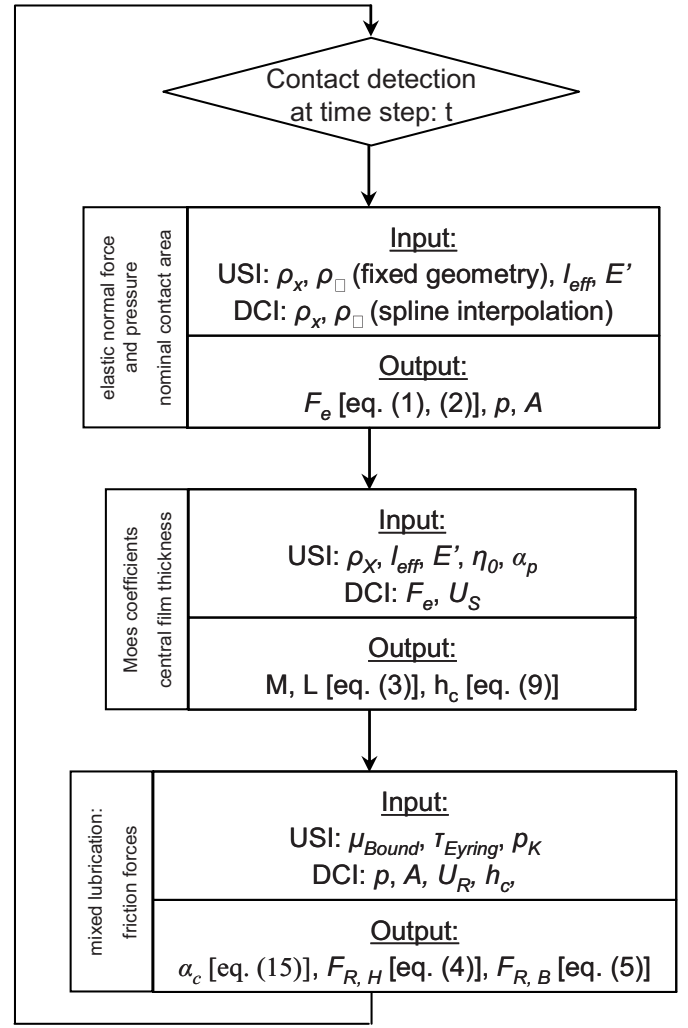

Fig. 2. One-dimensional EHL procedure (USI: User Input, DCI: Dynamically Calculated Input).

\subsection{Results and discussion}

In the following, some results and comments on the simulation of MBS systems with the one-dimensional mathematical model exposed above are presented. The first system is a highly-loaded pump lubricated by diesel fuel. Half an hour is needed to compute a complete rotation of the cam. In Figure 3, the evolution of the Moes' coefficients, the maximal contact pressure and the central lubrication film thickness are presented for $3 / 4$ of a rotation. These two last are normalised for confidentiality reasons. The values of $L$ and $M$ confirm the above assumption with a fluid behaviour near elastic isoviscous.

It shows an important influence of the contact load and validates the hypothesis used for the friction model. The main reasons for these results are of course the load properties but also the bad viscosity of the present fuel. It leads to very small contact film thicknesses (about $0.1 \mu \mathrm{m})$. The peak, present between $0.06 \mathrm{~s}$ and $0.07 \mathrm{~s}$, arises from a negative cam curvature, which has induced a modification of $\rho_{x}$ and of $U_{\mathrm{S}}$. Around the lower dead centre arise small values of $M$, which may lead to the rigid isoviscous behaviour. This phenomenon can occur with other operating conditions, for which the elasticity and the piezo-viscosity could be neglected. These results have not yet been confirmed by experimental studies but correspond to the initial predictions of the system behaviour. Another pump has been studied with this numerical model. It contains more contacts as the user-element


Fig. 3. EHL results from a heavily-loaded cam-roller contact for $3 / 4$ of a rotation.

is applied to study 15 contact locations. The MBS environment has been coupled with an external software which computes the hydraulic forces. The results are satisfactory for a simulation which takes about one hour for two rotations of the system and therefore allows optimizations and sensibility analysis of the complete system. However some of their contacts remain undefined and prevent the obtainment of definitive results, which motivates the elaboration of the next detailed contact model.

\section{Three-dimensional contact solution}

The objective of the following model is to compute 3D accurate representations of the fluid behaviour and the contact pressure distribution of contacts between halfspaces. The restrictions are identical to those from the previous contact model concerning the MBS environment. A coupled solution of the Reynolds and the Boussinesq equations is in this case required. This task is still under development and only a concept for the Boussinesq equation for dry contacts is investigated here.

\subsection{Dry contact}

The following numerical model is based on the elasticity theory for half-spaces [12]. The Boussinesq equation

$$
d(x, y)=\frac{2}{\pi E^{\prime}} \iint_{A} \frac{p\left(x^{\prime}, y^{\prime}\right) \mathrm{d} x^{\prime} \mathrm{d} y^{\prime}}{\sqrt{\left(x-x^{\prime}\right)^{2}+\left(y-y^{\prime}\right)^{2}}}
$$




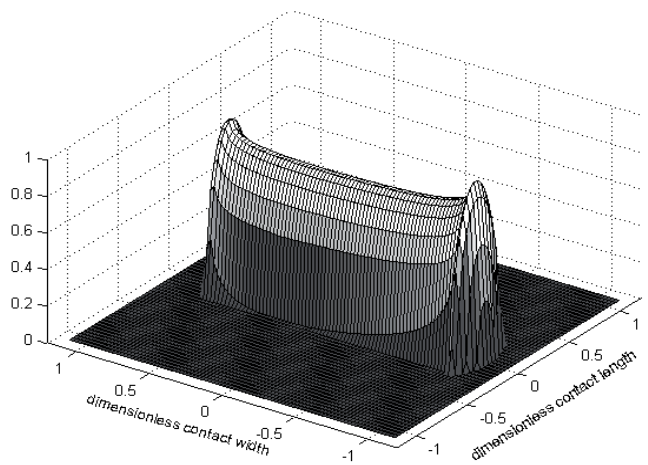

Fig. 4. Dimensionless pressure distribution in a line contact.

is solved to compute the pressure distribution for all time steps. The contour of the contacting bodies is discretised and the mutual approach $d(x, y)$ or the intersection volume is evaluated in a reference system associated with the contact plane. Equation (6) is then inverted in a discrete form and the solution is achieved as soon as the real contact area, often smaller than the intersection surface, is obtained. Therefore, an iterative process is performed on the pressure distribution [13]. Equation (6) may also be solved with a direct method as the Jacobi relaxation [6]; see Appendix C. Excellent results were obtained with both methods for a low consumption of computation time. The second one is however chosen according to a better computing time and to its involvement in the fully lubricated contact calculations. Some comparisons with well-known contact configurations as the Hertzian or line contacts (Fig. 4) have been done in order to validate the numerical model (error smaller than 1\%).

An improvement of this numerical model may be investigated: the influence of friction on the contact normal pressure distribution. The elasticity theory must be considered in more details. The simultaneous solution of normal contact and the friction or the micro-slip phenomena results from the inversion of the following equivalent equation:

$$
\tilde{A}\left[\begin{array}{l}
q_{x} \\
q_{y} \\
p
\end{array}\right]=\left[\begin{array}{l}
\delta_{x}-s_{x} \\
\delta_{y}-s_{y} \\
d(x, y)
\end{array}\right]
$$

where $\tilde{A}$ is a defined matrix [14], $\delta$ the tangential displacement and $s$ the sliding distance. The solution of this equation provides, however, some difficulties for the MBS environment, e.g. the measurement of tangential displacements or the storage of contact node positions from a dynamic contact plane. Only the sliding phenomenon can be calculated without reducing the efficiency of the above contact model. Therefore, the tangential component is related to the normal one by the Coulomb's friction coefficient and only the third component of the Equation (7) is solved.
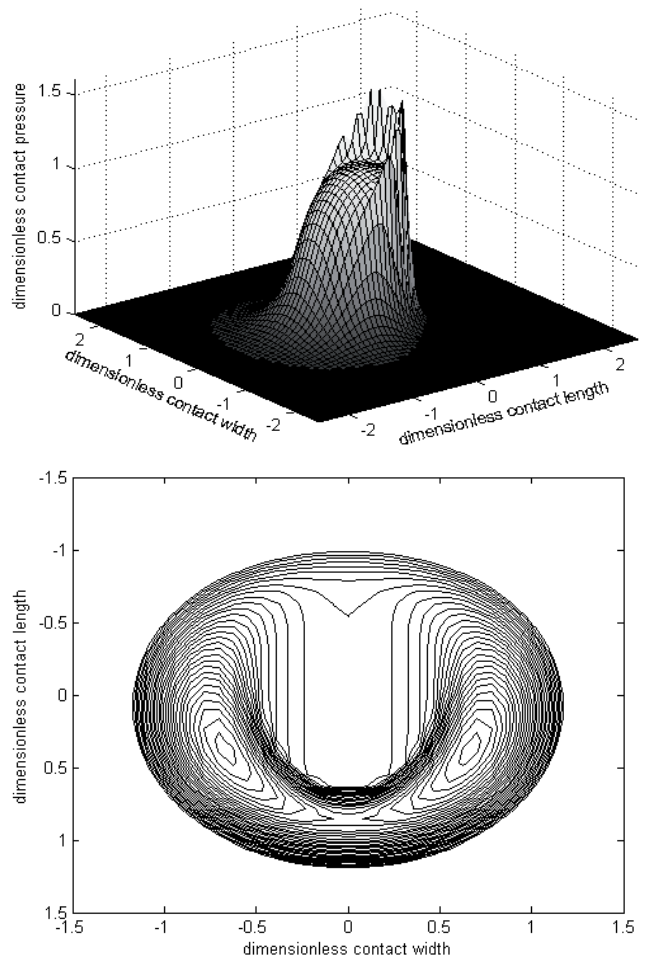

Fig. 5. Dimensionless pressure distribution and contour plot of the dimensionless film thickness in an EHL point contact.

\subsection{Discussion of the EHL contact problem}

In the following, some comments and perspectives are outlined for the improvement of the above numerical model considering fully lubricated contacts. The fluid-structure interaction arises from the coupling of the elasticity theory and the Reynolds equation. Two methods are distinguished for the present MBS model: the inverse method $[3,4]$ and the multi-grid method [6]. The two methods have an iterative process with specific convergence parameters. However the inverse method is restricted to highly loaded cases, which reduce its applicability. Moreover its convergence is semi-empirical and therefore may endanger the entire convergence of the MBS simulation. The direct method has been then chosen and firstly adapted to the MBS environment to solve EHD point contacts with smooth surfaces in order to test the stability and the calculating duration (Fig. 5). The numerical model has been compared and validated with known numerical solutions. An adaptation to line contacts and an improvement concerning the consideration of the roughness and the mixed lubrication are nowadays in progress.

Concerning the roughness, many studies have been done to improve and replace the well-known Greenwood/Tripp contact model, e.g. fractal distribution of asperity heights $[15,16]$. However, these models are still insufficient and the measurement of their characteristic parameters is not practicable for industrial applications. An experimental-based model is therefore suggested for the present numerical implementation. 


\section{Conclusion}

A simplified one-dimensional EHL contact model for cam contacts is proposed. It provides an approximation of the central film thickness and the friction behaviour and allows, due to the low consumption of computing time, optimisations or fast identifications of dynamical critical operating conditions. The user-subroutine is particularly appropriate for heavily loaded systems and can be rapidly adapted for a larger application range. A second 3D model is then introduced to overcome the simplified EHL results and provides a better description of the contact behaviour for future lifetime calculations. A comparison with experimental examinations will be conducted to validate the above numerical models.

\section{Appendix A: central lubricant film thickness for line contacts}

Given the Moes coefficients introduced in Equation (3), the contact behaviour may be evaluated following the asymptotic regimes:

- elastic isoviscous (EI): large $M, L=0$

- rigid isoviscous (RI): small $M, L=0$

- elastic piezoviscous (EP): large $M$, large $L$

- rigid piezoviscous (RP): small $M$, large $L$.

For each of these asymptotic regimes an asymptotic dimensionless film thickness may be defined depending on $M$ and $L$. For the line contact case, these are calculated as follows:

$$
\begin{aligned}
& H_{\mathrm{EI}}=2.621 M^{-1 / 5}, H_{\mathrm{RI}}=3 M^{-1} \\
& H_{\mathrm{EP}}=1.311 M^{-1 / 8} L^{3 / 4}, H_{\mathrm{RP}}=1.287 L^{2 / 3}
\end{aligned}
$$

The central lubrication film thickness results from a fit function of these asymptotic regimes:

$$
H_{\mathrm{C}}=\left[\left(H_{\mathrm{RI}}^{7 / 3}+H_{\mathrm{EI}}^{7 / 3}\right)^{3 s / 7}+\left(H_{\mathrm{RP}}^{-7 / 2}+H_{\mathrm{EP}}^{-7 / 2}\right)^{-2 s / 7}\right]^{1 / s}
$$

with $s=0.2\left[7+8 \exp \left(-2 H_{\mathrm{EI}} / H_{\mathrm{RI}}\right)\right]$ and

$$
H_{\mathrm{C}}=h_{\mathrm{C}} \rho_{x}\left(\frac{E^{\prime}}{\eta_{0} U_{\mathrm{S}} \rho_{x}}\right)^{1 / 2}
$$

\section{Appendix B: Generalized Greenwood/Tripp model}

Given a local nominal contact area $A_{\mathrm{m}}$, the contact force is determined by:

$$
F_{\text {asp }}=\frac{8 \sqrt{2} \pi}{15}\left(\eta_{\mathrm{GT}} \beta_{\mathrm{GT}} \sigma_{\mathrm{S}}\right)^{2} E^{\prime} \sqrt{\frac{\sigma_{\mathrm{S}}}{\beta_{\mathrm{GT}}}} F_{5 / 2}\left(\frac{h}{\sigma_{\mathrm{S}}}\right) A_{\mathrm{m}}
$$

and the according real contact area as:

$$
A_{\mathrm{asp}}=\pi^{2}\left(\eta_{\mathrm{GT}} \beta_{\mathrm{GT}} \sigma_{\mathrm{S}}\right)^{2} F_{2}\left(\frac{h}{\sigma_{\mathrm{S}}}\right) A_{\mathrm{m}}
$$

In Equations (10) and (11), the special functions $F_{2}$ and $F_{5 / 2}$ are defined as:

$$
F_{n}(\Phi)=\frac{1}{\sqrt{2 \pi}} \int_{\Phi}^{\infty}(s-\Phi)^{n} e^{-s^{2} / 2} \mathrm{~d} s
$$

The approximation of proportionality between the real contact area fraction and the local contact pressure is assumed, and the special functions are taken as identical for $h / \sigma_{\mathrm{S}} \in[0,1]$ so that the fraction of the real contact area is given by:

$$
\alpha_{\mathrm{asp}} \approx \frac{15 \sqrt{2} \pi}{16 E^{\prime}} \sqrt{\frac{\beta_{\mathrm{GT}}}{\sigma_{\mathrm{S}}}} p_{\mathrm{asp}}
$$

An integration of Equation (13) upon the overall macroscopic nominal contact surface leads to the real contact area of the macroscopic system:

$$
A_{\mathrm{asp}}=\left(\frac{15 \sqrt{2} \pi}{16 E^{\prime}} \sqrt{\frac{\beta_{\mathrm{GT}}}{\sigma_{\mathrm{S}}}}\right) F_{\mathrm{e}}=\frac{F_{\mathrm{e}}}{p_{K}}
$$

In Equation (14), a proportionality between the real contact area and the normal contact force is obtained. However, due to the heavy load, this proportionality can not be expected for the overall domain of definition of the pressure and particularly for $h \rightarrow 0$. In this case, the asperities merge and thus Equation (11) is no more applicable. Therefore, Equation (13) is generalized as follows:

$$
\alpha_{\mathrm{C}}(p)=\tanh \left(p / p_{K}\right)
$$

The proportionality is then maintained for low pressures (compared to $p_{\mathrm{K}}$ ) and $\alpha_{\mathrm{C}}$ reaches unity for high pressures. Typical values of $p_{\mathrm{K}}$ are in the range from $0.25 \%$ to $3 \%$ of $E^{\prime}$.

\section{Appendix C: 3D dry contact solution}

Equation (6) may be discretised and regarded as a linear system of equations.

$$
d_{i j}=\sum_{i^{\prime}, j^{\prime}} k_{i i^{\prime} j j^{\prime}} p_{i^{\prime} j^{\prime}}
$$

In Equation (16), $k$ are influence coefficients defined by:

$$
\begin{aligned}
k_{i i^{\prime} j j^{\prime}}= & \frac{2}{\pi E^{\prime}}\left\{\left|x_{\mathrm{p}}\right| \arcsin \left(\frac{y_{\mathrm{p}}}{x_{\mathrm{p}}}\right)+\left|y_{\mathrm{p}}\right| \arcsin \left(\frac{x_{\mathrm{p}}}{y_{\mathrm{p}}}\right)\right. \\
& -\left|x_{\mathrm{m}}\right| \arcsin \left(\frac{y_{\mathrm{p}}}{x_{\mathrm{m}}}\right)-\left|y_{\mathrm{p}}\right| \arcsin \left(\frac{x_{\mathrm{m}}}{y_{\mathrm{p}}}\right) \\
& -\left|x_{\mathrm{p}}\right| \arcsin \left(\frac{y_{\mathrm{m}}}{x_{\mathrm{p}}}\right)-\left|y_{\mathrm{m}}\right| \arcsin \left(\frac{x_{\mathrm{p}}}{y_{\mathrm{m}}}\right) \\
& \left.+\left|x_{\mathrm{m}}\right| \arcsin \left(\frac{y_{\mathrm{m}}}{x_{\mathrm{m}}}\right)+\left|y_{\mathrm{m}}\right| \arcsin \left(\frac{x_{\mathrm{m}}}{y_{\mathrm{m}}}\right)\right\}
\end{aligned}
$$


where

$$
\begin{aligned}
& x_{\mathrm{p}}=x_{i}-x_{i^{\prime}}+u_{x} / 2, x_{\mathrm{m}}=x_{i}-x_{i^{\prime}}-u_{x} / 2 \\
& y_{\mathrm{p}}=y_{j}-y_{j^{\prime}}+u_{y} / 2, y_{\mathrm{m}}=y_{j}-y_{j^{\prime}}-u_{y} / 2
\end{aligned}
$$

They represent the local displacement or deformation due to a pressure distribution on the complete mesh. In the followings, two methods are proposed for the solution. Equation (16) is first considered in a general dimensionless form:

$$
D_{i j}=\sum_{i^{\prime}, j^{\prime}} K_{i i^{\prime} j j^{\prime}} P_{i^{\prime} j^{\prime}}
$$

The first method consists in inverting the system with the $\mathrm{LU}$ decomposition. As the real contact area is not known, the procedure starts by considering an overestimated contact area. The inversion of Equation (6) results first in a composition of negative and positive contact pressures. It means that the positive pressure distribution does not correspond to the real one, and confirms that the contact area is overestimated. For the next iteration, negative pressures are set to zero, reducing the considered contact area and a new inversion is performed. This last iteration is then repeated until only positive pressures are obtained.

The second proposed method is the Jacobi relaxation. Given an approximated solution $\tilde{P}_{i j}$ of the real pressure distribution, the convergence of Equation (19) may be obtained as soon as the residual $r_{i j}$ defined in Equation (20) tends to zero.

$$
r_{i j}=D_{i j}-\sum_{i^{\prime}, j^{\prime}} K_{i i^{\prime} j j^{\prime}} \tilde{P}_{i^{\prime} j^{\prime}}
$$

Therefore a correction is applied to the approximated solution for each iteration and the new solution is given by:

$$
\bar{P}_{i j}=\tilde{P}_{i j}+w \Lambda_{i j}
$$

where $w$ is an underrelaxation factor (between 0 and 1) and $\Lambda_{i j}$ defined as follow when considering the mesh size as constant in both directions:

$$
\begin{aligned}
\Lambda_{i j} & =r_{i j} / K_{0,0} \\
\text { with } K_{\psi, \phi} & =K_{i i^{\prime}=i \pm \psi} j j^{\prime}=j \pm \phi
\end{aligned}
$$

However the correction factor defined in Equation (22) generates instability as the influence coefficients matrix only contains non-zero values. Indeed local corrections in the pressure distribution affect too largely the complete deformation and endanger the general convergence. A distributive relaxation is therefore advised [6], where additional corrections are applied to neighbouring mesh nodes. As a result, the influence of the correction on the overall deformation decays with increasing distance. In the case of a 2D dry contact, a second order distribution may be applied. The correction is then defined by:

$$
\Lambda_{i j}=r_{i j} /\left(K_{0,0}-K_{0,1} / 2-K_{1,0} / 2\right)
$$

and the new pressure distribution by:

$$
\bar{P}_{i j}=\tilde{P}_{i j}+w\left(\Lambda_{i j}-\frac{1}{4}\left(\Lambda_{i-1 j}+\Lambda_{i+1 j}+\Lambda_{i j-1}+\Lambda_{i j+1}\right)\right)
$$

\section{References}

[1] H. Moes, Lubrication and beyond, Utwente lecture notes code 115531,2000 , p. 366

[2] B.J. Hamrock, D. Dowson, Isothermal elastohydrodynamic lubrication of point contacts, Part 2, ellipticity parameter results, ASME J. Tribol. 98 (1976) 375-383

[3] D. Dowson, G.R. Higginson, A numerical solution to elastohydrodynamic problem, J. Mech. Eng. Sci. 1 (1959) 615

[4] H.P. Evans, R.W. Snidle, The elastohydrodynamic lubrication of point contacts at heavy loads, Proc. R. Soc. Lond. A 382 (1982) 183-199

[5] K.P. Hou, D. Zhu, S.Z. Wen, An inverse solution to the point contact EHL problem under heavy loads, ASME J. Tribol. 109 (1987) 432-436

[6] A.A. Lubrecht, C.H. Venner, Multilevel methods in lubrication, Amsterdam, Elsevier, 2000

[7] H. Hertz, Über die Berührung fester elastischer Körper und über die Härte, Verhandlungen des Vereins zur Beförderung des Gewerbefleißes, Leipzig, 1882, pp. 449463

[8] K. Kunert, Spannungsverteilung im Halbraum bei elliptischer Flächenpres-sungsverteilung über einer rechteckigen Druckfläche, Forschung Gebiet Ingenieur-Wesens 27 (1961) 165-174

[9] J.A. Greenwood, J.H. Tripp, The contact of two nominally flat rough surfaces, Proc. Instn. Mech. Engrs. 185 (1971) 625-633

[10] M. Teodorescu, D. Taraza, N.A. Henein, Simplified elastohydrodynamic friction model of the cam-tappet contact, SAE paper 2003-01-0985, 2003

[11] J. Müllers, Tribologische Modelle zur Einbindung in Mehrkörpersimulations-tools, Robert Bosch GmbH, 2005

[12] A.E.H. Love, A treatise on the mathematical theory of elasticity, Cambridge university press, 4th edition, 1927

[13] N. Ahmadi, L.M. Keer, T. Mura, Non-Hertzian contact stress analysis for an elastic half-space - normal and sliding contact, Int. J. Solids Structures 19 (1983) 357-373

[14] S. Björklund, S. Andersson, A numerical method for real elastic contacts subjected to normal and tangential loading, Elsevier, Wear 179 (1994) 117-122

[15] A. Majumdar, B. Bhushan, Role of fractal geometry in roughness characterization and contact mechanics of surfaces, ASME J. Tribol. 112 (1990) 205-216

[16] K. Willner, Elasto-plastic normal contact of threedimensional fractal surfaces using halfspace theory, ASME J. Tribol. 126 (2004) 28-33 